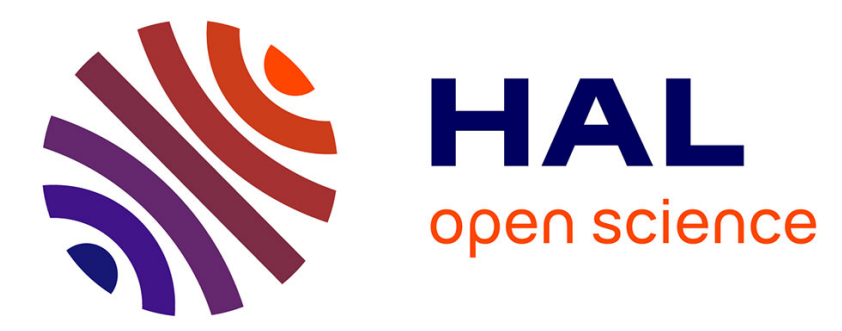

\title{
A Compact AWPC Antenna for DOA estimation
}

Tran Thi Thuy-Quynh, Nguyen Linh-Trung, Phan Anh, Karim Abed-Meraim

\section{To cite this version:}

Tran Thi Thuy-Quynh, Nguyen Linh-Trung, Phan Anh, Karim Abed-Meraim. A Compact AWPC Antenna for DOA estimation. nternational Symposium on Communications andInformation Technologies (ISCIT), Oct 2012, Gold Coast, Australia. hal-01002328

\section{HAL Id: hal-01002328 \\ https://hal.science/hal-01002328}

Submitted on 13 Jan 2015

HAL is a multi-disciplinary open access archive for the deposit and dissemination of scientific research documents, whether they are published or not. The documents may come from teaching and research institutions in France or abroad, or from public or private research centers.
L'archive ouverte pluridisciplinaire $\mathbf{H A L}$, est destinée au dépôt et à la diffusion de documents scientifiques de niveau recherche, publiés ou non, émanant des établissements d'enseignement et de recherche français ou étrangers, des laboratoires publics ou privés. 


\title{
A Compact AWPC Antenna for DOA estimation
}

\author{
Tran Thi Thuy-Quynh*, Nguyen Linh-Trung*, Phan Anh*, and Karim Abed-Meraim ${ }^{\dagger}$ \\ *Faculty of Electronics and Telecommunications, VNU University of Engineering and Technology, Hanoi, Vietnam \\ $\dagger$ Department of Image and Signal Processing, Telecom ParisTech, Paris, France \\ Email: quynhttt@vnu.edu.vn, linhtrung@vnu.edu.vn, phananh@ rev.org.vn, karim.abed@telecom-paristech.fr
}

\begin{abstract}
Antennas without phase center (AWPC) have been used for Direction-of-Arrival estimation. However, these structures, being symmetric, always face the whole-space ambiguity problem. This paper proposes a new asymmetric AWPC structure for DOA estimation that is able to resolve the ambiguity in the whole space. In addition, the structure is optimized in such a way that it is compact and isotropic. Simulated results indicate the effectiveness of the proposed structure.
\end{abstract}

Keywords- Direction of Arrival (DOA), Antenna without Phase Center (AWPC), Multiple Signal Classification (MUSIC), Cramer-Rao Bound (CRB).

\section{INTRODUCTION}

The fast development of high speed wireless communication systems has a strong connection with smart antenna technology in which direction-of-arrival (DOA) estimation and beamforming are two major techniques. A class of antennas without phase center (AWPC) was introduced in [1] with four dipoles $A, B, C$ and $D$, as shown in Fig. 1(a).

AWPC has been used for multiple source DOA estimation wherein there are less number of sensors than that of sources. In [2], a linear-phase- pattern AWPC prototype, and, in [3], another prototype of nonlinear-phase-pattern AWPC as a sensor in a Uniform Circular Array (UCA) was used. These works however face the problem of ambiguity, which is the similarity of two or more steering vectors corresponding to widely separated directions in the array manifold. Then, array geometries of AWPC have been considered in [4] in which some parameters of AWPC are optimized to increase accuracy and resolve ambiguity problem for arbitrary estimators. However, it only helps resolve half-space ambiguity, that is, no ambiguity occurs in the range $[-\pi / 2, \pi / 2]$. Whole-space ambiguity remains; i.e., in the range $[-\pi, \pi]$.

The above papers only focus on the AWPCs which have symmetric dipole couples; that is, $d_{1}=d_{2}$ and $d_{3}=d_{4}$ as shown in Fig. 1(a). The problem of ambiguity poses the following question: How to resolve the whole-space ambiguity which is inherent in the symmetric AWPC? The problem leads us to seek for a solution in which the sensors in the AWPC are arranged asymmetrically. We revert to calculating the electric field of such geometrical configuration for AWPC and observe that the AWPC is ambiguity-free in the whole space, if at least one of the two dipole couples is asymmetric. Based on these observations, we optimize the asymmetric AWPC with the constraint that the size of the antenna is as small as possible. This leads us to investigate in this paper the problem of how to design a compact AWPC which is free of ambiguity in the whole space.

In addition, we will take into account of two other desired properties for the AWPC: negligible mutual coupling and isotropic. Mutual coupling is a phenomenon that antenna elements/sensors in the array interact with each other if they are arranged nearly each other. Mutual coupling decreases the accuracy of estimate DOAs, and hence it is desired to reduce such an effect. It is well known in antenna theory that the effect of mutual coupling can be reduced remarkably if the distance between two arbitrary antenna sensors is larger than $\lambda / 2$. Isotropic array describes the antenna geometries which have uniform performance over the whole field of view [5]. Having an isotropic AWPC allows us to estimate the DOA of sources arriving at the AWPC from any direction.

The paper is organized as follows. Section II presents the asymmetric AWPC structure and the array factor (AF). Section III introduces the proposed asymmetric AWPC which has a compact size and negligible effect of mutual coupling. Section IV analyzes the antenna geometries optimized for having the best ambiguity-free level and isotropic characteristic. Section $\mathrm{V}$ provides a numerical example to demonstrate the effectiveness of the optimized asymmetric AWPC. Section VI concludes the paper.

\section{Asymmetric AWPC Structure}

The proposed asymmetric AWPC is illustrated in Fig. 1(b), with four dipoles $A, B, C$ and $D$. The structure is asymmetric in the sense that $d_{1} \neq d_{2}$ and $d_{3} \neq d_{4}$. According to antenna theory, the total electric field of the sensors in the antenna array is expressed by

$$
E(\theta)=-\frac{j k}{4 \pi} \frac{e^{-j k R_{0}}}{R_{0}}\left|I_{0}\right| \beta(\theta),
$$

where $\theta$ is the direction of propagation, $k$ is wave number, $R_{0}$ is distance between antenna array and source, $\left|I_{0}\right|$ is amplitude of the current of each sensor, and $\beta(\theta)$ is the array factor.

In asymmetric AWPC case, $\beta(\theta)$ is given by

$$
\begin{aligned}
\beta(\theta) & =e^{j \psi_{1}} e^{-j k d_{1} \sin \theta}+e^{j \psi_{2}} e^{j k d_{2} \sin \theta} \\
& +e^{j \psi_{3}} e^{j k d_{3} \cos \theta}+e^{j \psi_{4}} e^{-j k d_{4} \cos \theta},
\end{aligned}
$$

where $\psi_{1}=0^{\circ}, \psi_{2}=180^{\circ}, \psi_{3}=90^{\circ}$, and $\psi_{4}=270^{\circ}$ are phases of the currents of sensors $A, C, B$, and $D$, respectively.

Under the above conditions for locations of the sensors and the feeding points, the amplitude pattern, $G(\theta)$, and the phase 


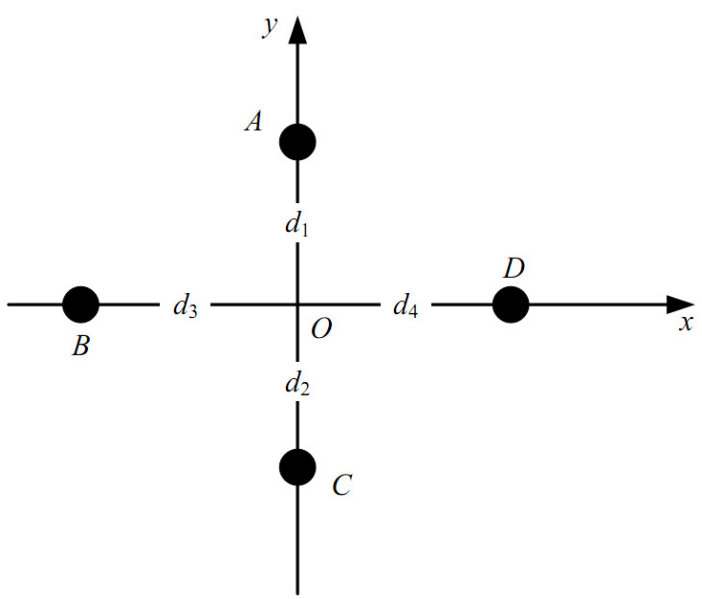

(a) Symmetric: $d_{1}=d_{2}, d_{3}=d_{4}$

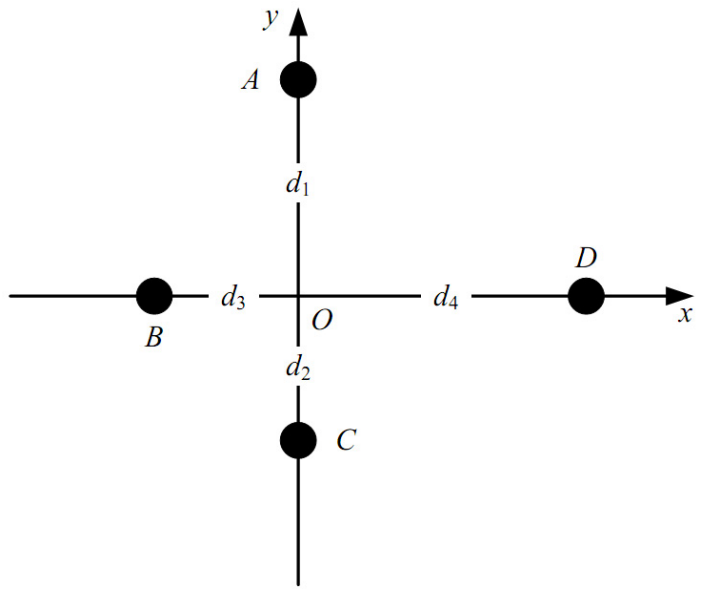

(b) Asymmetric: $d_{1} \neq d_{2}, d_{3} \neq d_{4}$

Fig. 1. AWPC structures.

pattern, $\Phi(\theta)$, of the non-symmetric AWPC are given by

$$
\begin{aligned}
& G(\theta)=\sqrt{\Re^{2}\{\beta(\theta)\}+\Im^{2}\{\beta(\theta)\}}, \\
& \Phi(\theta)=\angle \beta(\theta),
\end{aligned}
$$

where $\angle$ denotes the phase of a complex number, and

$$
\begin{aligned}
\Re\{\beta(\theta)\} & =-2 \sin \left[\frac{k}{2}\left(d_{1}+d_{2}\right) \sin \theta\right] \sin \left[\frac{k}{2}\left(d_{1}-d_{2}\right) \sin \theta\right] \\
& -2 \sin \left[\frac{k}{2}\left(d_{3}+d_{4}\right) \cos \theta\right] \cos \left[\frac{k}{2}\left(d_{3}-d_{4}\right) \cos \theta\right] \\
\Im\{\beta(\theta)\} & =-2 \sin \left[\frac{k}{2}\left(d_{1}+d_{2}\right) \sin \theta\right] \cos \left[\frac{k}{2}\left(d_{1}-d_{2}\right) \sin \theta\right] \\
& -2 \sin \left[\frac{k}{2}\left(d_{3}+d_{4}\right) \cos \theta\right] \sin \left[\frac{k}{2}\left(d_{3}-d_{4}\right) \cos \theta\right]
\end{aligned}
$$

It can be observed that if $d_{1} \neq d_{2}$ and/or $d_{3} \neq d_{4}$ then

$$
\begin{gathered}
G\left(\theta \pm \frac{\pi}{2}\right) \neq G(\theta) \\
G(\theta \pm \pi) \neq G(\theta),
\end{gathered}
$$

Eq. (7) indicates that the asymmetric AWPC has no half-space ambiguity (also denoted as $\pi / 2$-ambiguity) and (8) shows that it also has no whole-space ambiguity (also called $\pi$ ambiguity) [4].

\section{New COMPaCt Asymmetric AWPC With No $\pi$-Ambiguity AND Negligible Mutual Coupling}

It is desired to reduce the mutual coupling in the AWPC. The larger the distances between the array sensors, the lower the coupling. It is well known that mutual coupling will decrease remarkably if the distance is larger than $\lambda / 2$. Thus, there is a design trade-off between reducing the size of the AWPC and experiencing negligible mutual coupling. The following geometrical configuration is then proposed:

$$
\left(d_{1}, d_{2}, d_{3}, d_{4}\right)=(\lambda / 4, \lambda / 4, \sqrt{3} \lambda / 4,(\sqrt{3} / 4+\Delta d) \lambda) .
$$

In this configuration, sensors $\mathrm{A}, \mathrm{B}$ and $\mathrm{C}$ are equally spaced at a distance of $\lambda / 2$, for negligible mutual coupling between these sensors. The additional scaling factor $(\Delta d)>0$ is added to maintain the asymmetric characteristic of the AWPC. Therefore, the AWPC is considered as a compact AWPC.

Fig. 2(a) plots the ambiguity checking function (ACF) of the symmetric AWPC with $\Delta d=0$ and Fig. 2(b) plots the ACF of the asymmetric AWPC with $\Delta d=1-\sqrt{3} / 4>0$. More information on the ACF is deferred until Section IV-B. For $\theta_{1} \neq \theta_{2}$, Fig. 2(a) shows that $\gamma\left(\theta_{1}, \theta_{2}\right)=0$ at $\left(\theta_{1}, \theta_{2}=\theta_{1} \pm \pi\right)$ while Fig. 2(b), we observe that $\gamma\left(\theta_{1}, \theta_{2}\right) \gg 0$. Therefore, the proposed asymmetric AWPC structure in (9) has no $\pi$ ambiguity and negligible mutual coupling.

\section{ISOTROPIC AWPC}

This section further investigates on the optimal value of $\Delta d$ so that the proposed compact AWPC in (9) is isotropic.

\section{A. Data Model}

Assume that elevation angle is equal to $90^{\circ}$, consider $D$ uncorrelated, narrowband, zero-mean Gaussian sources, impinging on the non-symmetric AWPC: $s_{1}(t), s_{2}(t), \ldots, s_{D}(t)$. The antenna is rotated in $M$ steps in the clockwise direction. At step $m$, for $m=0, \ldots, M-1$, the received signal is modeled as

$$
x_{m}(t)=\sum_{i=1}^{D} s_{i}(t) G\left(\theta_{i}+m \Delta \theta\right) e^{j \Phi\left(\theta_{i}+m \Delta \theta\right)}+n_{m}(t),
$$

where $\theta_{i}$ is the incident angle of the $i$-th source, $\Delta \theta$ is the antenna rotation angle, and $n_{m}(t)$ is the spatially zero-mean white Gaussian noise with variance of $\sigma^{2}$ and is statistically independent of the sources. In matrix form, the data model becomes

$$
\mathbf{x}(t)=\mathbf{A}(\theta) \mathbf{s}(t)+\mathbf{n}(t),
$$

where $\mathbf{s}(t)=\left[s_{1}(t), s_{2}(t), \ldots, s_{D}(t)\right]^{T}$ is the source vector, $\mathbf{n}(t)=\left[n_{1}(t), n_{2}(t), \ldots, n_{M}(t)\right]^{T}$ is the noise vector, $\mathbf{x}(t)=$ $\left[x_{1}(t), x_{2}(t), \ldots, x_{M}(t)\right]^{T}$ is the received vector, and $\mathbf{A}(\theta)$ is the steering matrix defined by

$$
\mathbf{A}(\theta)=\left[\mathbf{a}\left(\theta_{1}\right), \mathbf{a}\left(\theta_{2}\right), \ldots, \mathbf{a}\left(\theta_{D}\right)\right],
$$




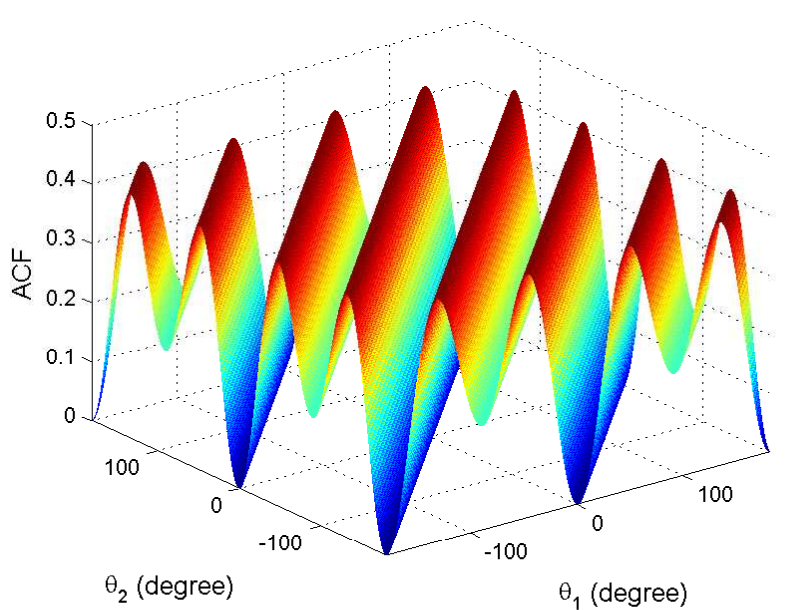

(a) Symmetric: $\Delta d=0$

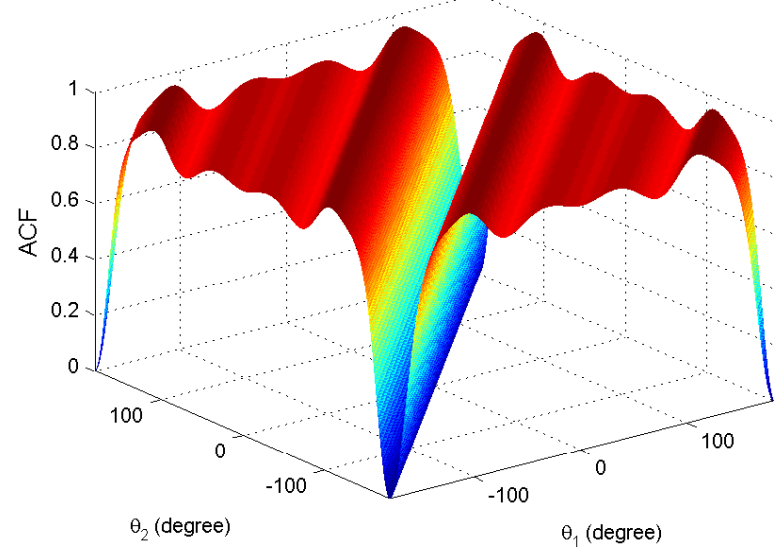

(b) Asymmetric: $\Delta d=1-\frac{\sqrt{3}}{4}$

Fig. 2. Ambiguity of symmetric and asymmetric AWPC; no ambiguity shown in 2(b).

In (12), $\mathbf{a}\left(\theta_{i}\right)$ is the steering vector associated with the $i$-th source and is given by

$$
\mathbf{a}\left(\theta_{i}\right)=\left[\begin{array}{c}
G\left(\theta_{i}\right) e^{j \Phi\left(\theta_{i}\right)} \\
G\left(\theta_{i}+\Delta \theta\right) e^{j \Phi\left(\theta_{i}+\Delta \theta\right)} \\
\vdots \\
G\left(\theta_{i}+(M-1) \Delta \theta\right) e^{j \Phi\left(\theta_{i}+(M-1) \Delta \theta\right)}
\end{array}\right] .
$$

The spatial covariance matrix of the output vector is expressed as

$$
\mathbf{R}_{\mathbf{x}}=\mathbf{E}\left\{\mathbf{x}(t) \mathbf{x}^{H}(t)\right\}=\mathbf{A R}_{\mathbf{s}} \mathbf{A}^{H}+\sigma^{2} \mathbf{I},
$$

where $\mathbf{E}\{\cdot\}$ denotes the statistical expectation, and $\mathbf{R}_{\mathbf{S}}$ is the source covariance matrix.

\section{B. Ambiguity-Free Level}

In [4], the ambiguity checking function is used for measuring the similarity relationship between two arbitrary steering

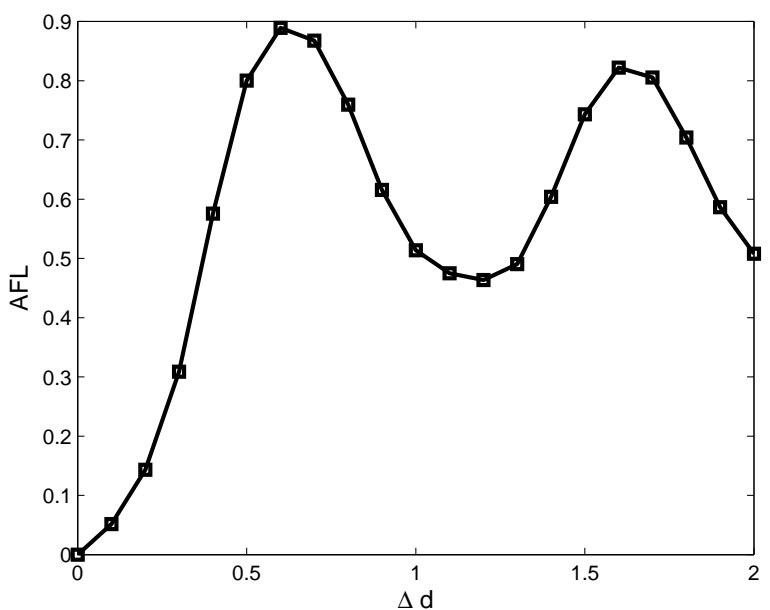

Fig. 3. AFL versus $\Delta d$

vectors $\mathbf{a}\left(\theta_{1}\right)$ and $\mathbf{a}\left(\theta_{2}\right)$ at directions $\theta_{1}$ and $\theta_{2}$, and is defined as:

$$
\gamma\left(\theta_{1}, \theta_{2}\right) \triangleq 1-\frac{\left|\mathbf{a}^{H}\left(\theta_{1}\right) \mathbf{a}\left(\theta_{2}\right)\right|^{2}}{\left\|\mathbf{a}\left(\theta_{1}\right)\right\|^{2}\left\|\mathbf{a}\left(\theta_{2}\right)\right\|^{2}}
$$

If $\mathbf{a}\left(\theta_{1}\right)$ and $\mathbf{a}\left(\theta_{2}\right)$ are co-linear then $\gamma\left(\theta_{1}, \theta_{2}\right)=0$ and if they are orthogonal, meaning $\left|\mathbf{a}^{H}\left(\theta_{1}\right) \mathbf{a}\left(\theta_{2}\right)\right|=0$, then $\gamma\left(\theta_{1}, \theta_{2}\right)=$ 1 . The array geometry has no ambiguity if $0 \ll \gamma\left(\theta_{1}, \theta_{2}\right) \leq 1$.

In this paper, we further define another measure- the Ambiguity-Free Level (AFL)-, in order to quantify the depth of ambiguity error, such as $\pi$-ambiguity in Fig. 2(a), as given by

$$
\eta(\Delta d) \triangleq \min _{\theta_{1}, \theta_{2}} \gamma\left(\theta_{1}, \theta_{2} ; \Delta d\right) \quad \text { s.t. } \epsilon_{1}<\left|\theta_{1}-\theta_{2}\right|<\epsilon_{2}
$$

where $\epsilon_{1}$ and $\epsilon_{2}$ are two predefined thresholds (in rads) for the optimization constraint. A higher value of $\eta(\Delta d)$ indicates a lower ambiguity error. When $\eta(\Delta d))=0$, ambiguity occurs.

Fig. 3 plots $\eta(\Delta d)$ constrained by $\left|\theta_{1}-\theta_{2}\right|>\lambda / 2$ of the proposed asymmetric AWPC. We can observe that $\eta(\Delta d)$ reaches its maximum at $\Delta d=0.6$, which means that this scaling factor is the best in terms of reducing the ambiguity in the proposed AWPC structure.

\section{Isotropic array}

In DOA estimation, the Cramer Rao Bound (CRB) function of a single source is a well-known bound that expresses the minimum achievable variance on estimating parameters of any unbiased estimator [6]. The antenna array is isotropic if its corresponding CRB is constant [7]. The CRB is defined as:

$$
\mathcal{C}(\theta)=\mathbf{J}^{-1},
$$




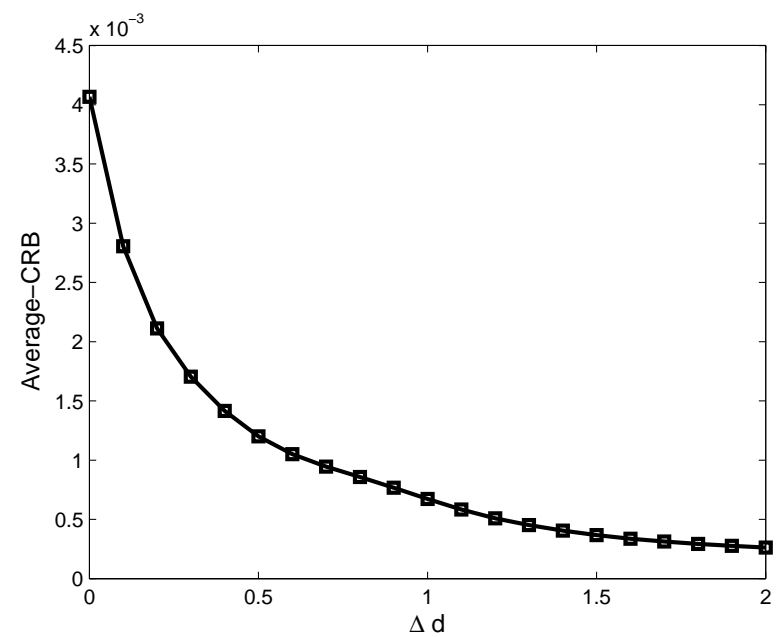

Fig. 4. Average-CRB versus $\Delta d$

where $\mathbf{J}$ is called the Fisher matrix. It has been shown in [4] that

$$
\begin{aligned}
\mathbf{J} & =N_{s} \cdot \operatorname{trace}\left\{\mathbf{R}_{\mathbf{x}}^{-1} \frac{\partial \mathbf{R}_{\mathbf{x}}}{\partial \theta} \mathbf{R}_{\mathbf{x}}^{-1} \frac{\partial \mathbf{R}_{\mathbf{x}}}{\partial \theta}\right\} \\
& =\frac{2 \mathrm{SNR}^{2}}{\left(1+\operatorname{SNR}|\mathbf{a}|^{2}\right)^{2}}\left[2\left(\Re\left(\mathbf{a}^{H} \dot{\mathbf{a}}_{\theta}\right)\right)^{2}\right. \\
& \left.+\left(1+\operatorname{SNR}|\mathbf{a}|^{2}\right)\left(|\mathbf{a}|^{2}|\dot{\mathbf{a}}|^{2}-\left|\mathbf{a}^{H} \dot{\mathbf{a}}_{\theta}\right|^{2}\right)\right],
\end{aligned}
$$

where $\dot{\mathbf{a}}_{\theta}=\partial \mathbf{a} / \partial \theta$. For simplicity, CRB is computed with $N_{s}=1$ only because the results for $N_{s}>1$ can be obtained by dividing the CRB values by $N_{s}$.

In general, one-dimensional linear arrays are non-isotropic and thus the performance of DOA estimation using these arrays often degrades considerably near the endfire [5]. The proposed AWPC structure is, however, two-dimensional and it is possible to obtain some $\Delta d$ so that this asymmetric AWPC is isotropic.

Next, we use numerical optimization to estimate this value of $\Delta d$. We select the range $(0,2]$ of $\Delta d$ for performing the optimization since we are interested only in AWPCs with a compact size. This is done based on the following two measures: averaged $C R B(\overline{\mathcal{C}})$ and margin $C R B\left(\epsilon_{\mathcal{C}}\right)$, defined as

$$
\begin{aligned}
\overline{\mathcal{C}}(\Delta d) & =\frac{1}{K} \sum_{\theta} \mathcal{C}(\theta, \Delta d) \\
\epsilon_{\mathcal{C}}(\Delta d) & =\max \mathcal{C}(\theta, \Delta d)-\min \mathcal{C}(\theta, \Delta d)
\end{aligned}
$$

Fig. 4 plots the averaged CRB of the proposed asymmetric AWPC structure. It indicates that $\overline{\mathcal{C}}$ decreases as $\Delta d$ increases. The larger $\Delta d$ value is, the higher the estimation accuracy is. This plot also shows that $\overline{\mathcal{C}}$ decreases strongly in the range $(0,1.4]$ and converge at very large $\Delta d$.

Fig. 5 plots the margin $\mathrm{CRB}$ of the proposed asymmetric AWPC structure. It can be seen that $\epsilon_{\mathcal{C}}$ stays constant in the range $\Delta d=(0,0.6]$ while it changes noticeably outside of this range.

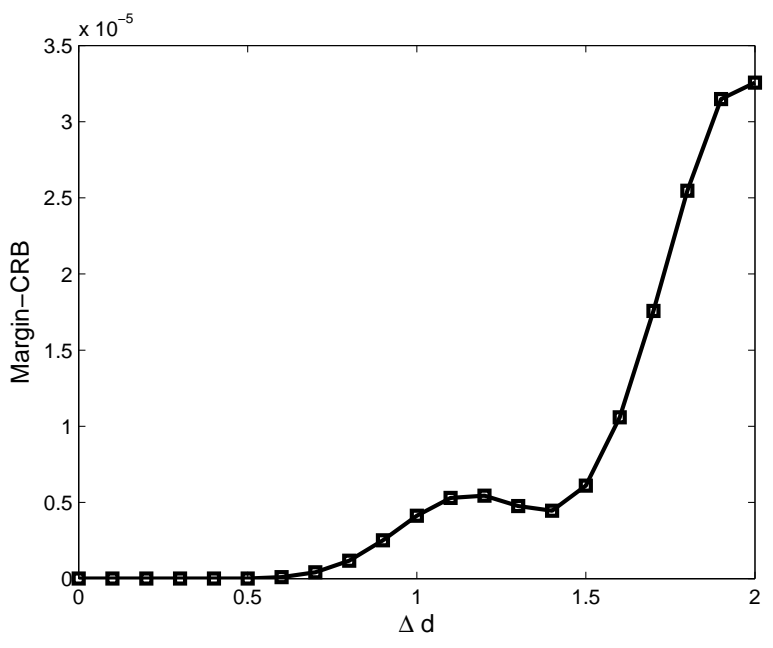

Fig. 5. Margin-CRB versus $\Delta d$

Based on above optimization it can be concluded that we should select the optimal scaling factor $\Delta d_{\text {opt }}=0.6$ for the proposed asymmetric AWPC structure in order to have it ambiguity-free, mutual-coupling negligible and compact.

\section{Numerical Simulations}

In this section, we provide a numerical example of DOA estimation that demonstrates the effectiveness of the proposed optimized asymmetric AWPC. Six sources are presented at azimuth $\left(-60^{\circ},-40^{\circ},-20^{\circ}, 20^{\circ}, 40^{\circ}, 60^{\circ}\right)$ and with a signal-to-noise ratio of $25 \mathrm{~dB}$. DOA estimation is based on the multiple-signal classification (MUSIC) algorithm (see [3] for more details). The MUSIC estimator is applied to 1000 random data snapshots. We test the following two AWPC structures:

1) Symmetric AWPC structure with

$$
\left(d_{1}, d_{2}, d_{3}, d_{4}\right)=(\lambda / 4, \lambda / 4, \sqrt{3} \lambda / 4, \sqrt{3} \lambda / 4) .
$$

2) Asymmetric AWPC structure with

$$
\left(d_{1}, d_{2}, d_{3}, d_{4}\right)=(\lambda / 4, \lambda / 4, \sqrt{3} \lambda / 4,(\sqrt{3} / 4+0.6) \lambda) .
$$

The results corresponding to symmetric and asymmetric structures are respectively shown in Figs. 6(a) and 6(b). The dashed lines present the original DOAs while the solid lines present the estimated DOA spectrum. The symmetric structure in Figs. 6(a) has steering vectors that are colinear at $\left(\theta_{1}, \theta_{2}=\theta_{1} \pm \pi\right)$, exhibiting the $\pi$-ambiguity error. Therefore, apart from the six origin peaks at $\left(-60^{\circ},-40^{\circ},-20^{\circ}, 20^{\circ}\right.$, $\left.40^{\circ}, 60^{\circ}\right)$, there are also 6 "ghost" peaks at $\left(-160^{\circ},-140^{\circ}\right.$, $-120^{\circ}, 120^{\circ}, 140^{\circ}, 160^{\circ}$ ).

The asymmetric structure in Figs. 6(b), with optimal scaling factor $\Delta d_{\mathrm{opt}}=0.6$, has steering vectors that are orthogonal at every $\left(\theta_{1}, \theta_{2}\right)$. Therefore, in whole-space, the MUSIC spectrum consists of only six peaks which are matched with the six original DOAs at $\left(-60^{\circ},-40^{\circ},-20^{\circ}, 20^{\circ}, 40^{\circ}, 60^{\circ}\right)$.

We remind that all simulation examples in this paper use $M=17$ and $\Delta \theta=2 \pi / M$ which are the optimal values 


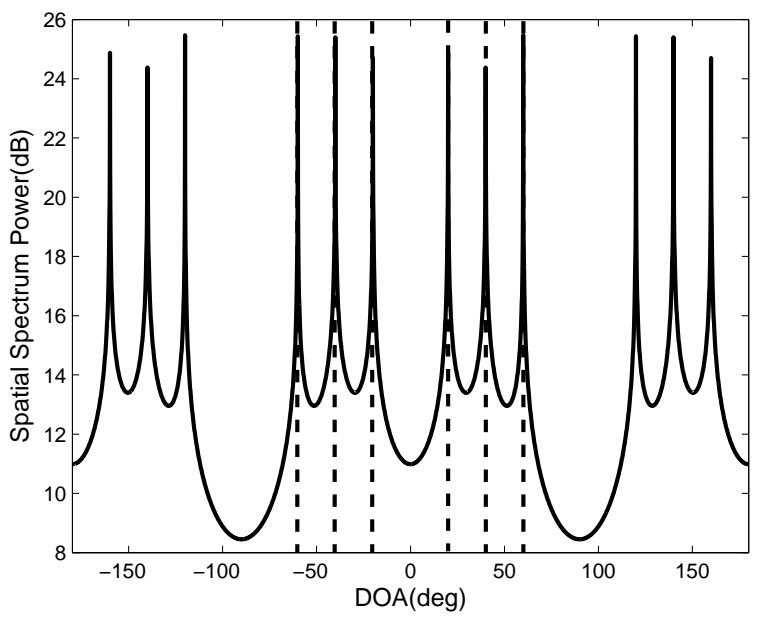

(a) Symmetric AWPC

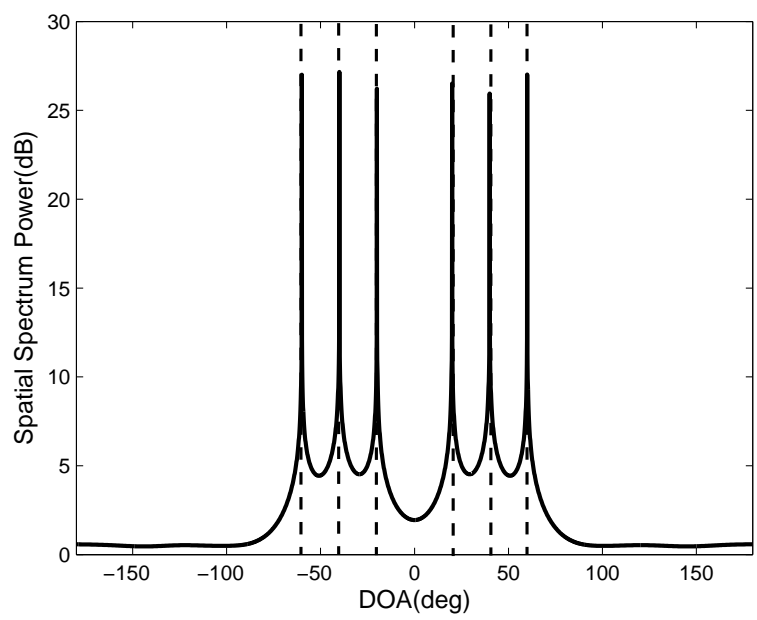

(b) Asymmetric AWPC

Fig. 6. MUSIC spectra for DOA estimation. $\Delta d_{\mathrm{opt}}=0.6$ for $6(\mathrm{~b})$.

found by [4] wherein a symmetric AWPC structure was used. However, as tested but not shown in this paper, these values can also be applied for the proposed asymmetric AWPC structure.

\section{CONCLusions}

In this paper we have resolved the whole-space ambiguity problem of symmetric AWPCs for DOA estimation. This is done by modifying the usual symmetric structure to an asymmetric one. In addition, we obtained the optimal scaling factor $\Delta d_{\mathrm{opt}}=0.6$ when targetting the asymmetric AWPC to be compact and isotropic.

\section{REFERENCES}

[1] P. Anh, Antennas without Phase Centers and their Applications in Radio Engineering, ser. Monograph, Wroclaw, Poland, 1986, no. 23, (ISSN: 0324-9328).
[2] P. Anh and Q. T. Cao, "DOA determination by using an antenna system without phase center and MUSIC algorithm," in Proc. IEEE Int. Symp. Antennas and Propagation Society, vol. 4, Washington DC, USA, July 2005, pp. 134-137.

[3] T. T. T. Quynh, P. P. Hung, P. T. Hong, T. M. Tuan, and P. Anh, "Directionof-arrival estimation using special phase pattern antenna elements in uniform circular array," in Int. Conf. Computational Intelligence and Vehicular System (CIVS 2010), 2010, pp. 138-141.

[4] T. T. T. Quynh, N. L. Trung, P. Anh, and K. Abed-Meraim, "On optimization of single-sensor antennas without phase center for DOA estimation," in 4th Int. Conf. Communications and Electronics (ICCE 2012), Hue, Vietnam, Aug. 2012, (accepted).

[5] U. Baysal and R. L. Moses, "On the geometry of isotropic arrays," IEEE Transactions on Signal Processing, vol. 51, no. 6, pp. 1469-1478, 2003.

[6] H. L. Van Trees, Optimum Array Processing - Part IV: Detection, Estimation, and Modulation Theory. John Wiley \& Sons, 2002.

[7] H. Gazzah and K. Abed-Meraim, "Optimum ambiguity-free isotropic antenna arrays," in Proc. IEEE Int. Conf. Acoustics, Speech and Signal Processing (ICASSP 2009), 2009, pp. 2157-2160. 